\title{
Long-term optical/IR variability of the Be/X-ray binary LS V +44 17/RX J0440.9+4431
}

\author{
P. Reig ${ }^{1}$, I. Negueruela ${ }^{2}$, J. Fabregat ${ }^{3}$, R. Chato ${ }^{3}$, and M. J. Coe ${ }^{4}$ \\ 1 IESL (FORTH) \& University of Crete, Physics Department, PO Box 2208, 71003 Heraklion, Crete, Greece \\ e-mail: pau@physics.uoc.gr \\ 2 Departamento de Física, Ingeniería de Sistemas y Teoría de la Señal, Universidad de Alicante, 03080 Alicante, Spain \\ 3 Observatorio Astronómico, Universitat de Valencia, 46071 Paterna-Valencia, Spain \\ ${ }^{4}$ School of Physics and Astronomy, The University, Southampton, SO17 1BJ, UK
}

Received 24 March 2005 / Accepted 6 June 2005

\begin{abstract}
We present the first long-term study of the optical counterpart to the X-ray pulsar RX J0440.9+4431/LS V +44 17. The data consist of optical spectroscopic and infrared photometric observations taken during the period 1995-2005. The infrared observations are the first published for this source. The results of our photometric and spectroscopic analysis show that RX J0440.9+4431/LS V +44 17 contains a moderately reddened, $E(B-V)=0.65 \pm 0.05, \mathrm{~B} 0.2 \mathrm{~V}$ star located at about $3.3 \mathrm{kpc}$. The $\mathrm{H} \alpha$ line consistently shows a double-peak profile varying from symmetric shape to completely distorted on one side ( $V / R$ phases). A correlation between the equivalent width of the $\mathrm{H} \alpha$ line and the infrared magnitudes is seen: as the $E W(\mathrm{H} \alpha)$ decreases the IR magnitudes become fainter. This long-term optical/IR variability is attributed to structural changes in the Be star's circumstellar disc. The observations include a recent decline in the circumstellar disc and subsequent recovery. We have witnessed the cessation of a global oscillation due to the decline of the circumstellar disc. If the present disc growth rate continues we predict the onset of another episode of $V / R$ variability by the end of 2006. We have investigated the typical time scales for disc variability of various $\mathrm{Be} / \mathrm{X}$-ray binaries and found a correlation with the orbital period. This correlation is hard to establish due to the difficulty in defining the exact duration of the various activity states, but it is seen both in the duration of the disc growth/dissipation phase and the value of the $\mathrm{H} \alpha$ equivalent width prior to the appearance of asymmetric profiles. These relationships provide further evidence for the interaction of the neutron star with the circumstellar disc of the Be star's companion and confirms the need of a fully developed disc for the $V / R$ variability to be observed.
\end{abstract}

Key words. stars: individual: RX J0440.9+4431, LS V +44 17 - X-rays: binaries - stars: neutron - stars: binaries: close stars: emission-line, Be

\section{Introduction}

LS V +4417 is a relatively bright $V=10.8$ B0 star that is associated with the X-ray source RX J0440.9+4431. RX J0440.9+4431/LS V +44 17 belongs to the subgroup of high-mass X-ray binaries known as Be/X-ray binaries. These systems consist of a neutron star orbiting a O9e-B2e star. The letter $e$ stands for emission, as instead of the normal photospheric absorption lines the optical spectra of Be stars display emission lines. Although helium and iron are occasionally seen in emission, the hydrogen lines, especially those of the Balmer series, constitute the signature for which Be stars are renowned (Porter \& Rivinius 2003). Strong infrared emission is another defining characteristic of Be stars. The origin of these two observational properties (emission lines and infrared excess) resides in a gaseous, equatorially concentrated circumstellar disc around the OB star. This disc acts as a reservoir of material for accretion on to the compact object. Although the ultimate cause of the Be phenomenon is still not known, it is believed to be related to the rapid rotation of these stars. Recent studies (Townsend et al. 2004) indicate that Be stars may rotate much closer to break-up velocity that previously thought.

The optical/IR information on LS V +44 17 is very scarce. It mainly comes from surveys of the Galactic Plane or catalogues prepared for specific space missions. However, no variability studies of this system have ever been reported. Interest in LS V +44 17 grew when the first evidence that it might be an X-ray binary came to light (Motch et al. 1997). RX J0440.9+4431 is an X-ray pulsar with a spin period of $202 \mathrm{~s}$ and belongs to the poorly studied group of persistent Be/X-ray binaries (Reig \& Roche 1999).

In this work we present the results of our monitoring programme on high-mass X-ray binaries for RX J0440.9+4431/LS V +44 17. We have performed a detailed analysis of its optical and infrared variability covering a period of almost 10 years. We have also made a comparative study of the long-term variability time scales of various $\mathrm{Be} / \mathrm{X}$-ray binaries. 
Table 1. Log of the spectroscopic observations.

\begin{tabular}{|c|c|c|c|c|c|c|c|}
\hline \multicolumn{8}{|c|}{ Red-end spectra } \\
\hline Date & $\begin{array}{c}\text { Telescope/ } \\
\text { observatory }\end{array}$ & $\begin{array}{c}\text { Dispersion } \\
\AA ̊ ̊ \\
\text { A/pixel }\end{array}$ & $\begin{array}{c}\text { Grating } \\
1 / \mathrm{mm}\end{array}$ & $\begin{array}{c}\text { Wavelength } \\
\AA\end{array}$ & MJD & $\begin{array}{c}E W(\mathrm{H} \alpha) \\
\AA\end{array}$ & $\log (V / R)^{a}$ \\
\hline 29-11-1995 & $1.0 \mathrm{~m} / \mathrm{ORM}$ & 1.07 & 1200 & $6100-6960$ & 50050.5 & $-9.3 \pm 0.4$ & 0.07 \\
\hline 28-02-1996 & $1.0 \mathrm{~m} / \mathrm{ORM}$ & 0.34 & 2400 & $6400-6750$ & 50141.5 & $-8.8 \pm 0.4$ & -0.25 \\
\hline $26-10-1997$ & $1.0 \mathrm{~m} / \mathrm{ORM}$ & 0.90 & 1200 & $5900-6800$ & 50747.6 & $-8.3 \pm 0.3$ & 0.00 \\
\hline 28-10-1997 & $1.0 \mathrm{~m} / \mathrm{ORM}$ & 0.34 & 2400 & $6380-6720$ & 50749.6 & $-8.3 \pm 0.4$ & -0.02 \\
\hline $20-07-2000$ & $1.3 \mathrm{~m} / \mathrm{SKI}$ & 1.04 & 1302 & $5550-7550$ & 51745.6 & $-4.4 \pm 0.2$ & -0.03 \\
\hline 05-10-2000 & $1.3 \mathrm{~m} / \mathrm{SKI}$ & 1.04 & 1302 & $5250-7290$ & 51822.6 & $-4.4 \pm 0.1$ & 0.04 \\
\hline 07-08-2001 & $1.3 \mathrm{~m} / \mathrm{SKI}$ & 1.04 & 1302 & $5660-7440$ & 52128.6 & $-0.2 \pm 0.1$ & 0.06 \\
\hline 12-09-2001 & $1.3 \mathrm{~m} / \mathrm{SKI}$ & 1.04 & 1302 & $5220-7190$ & 52164.6 & $-0.5 \pm 0.1$ & -0.03 \\
\hline 13-09-2001 & $1.3 \mathrm{~m} / \mathrm{SKI}$ & 1.04 & 1302 & $5230-7200$ & 52165.6 & $-0.5 \pm 0.1$ & -0.02 \\
\hline 08-10-2001 & $1.3 \mathrm{~m} / \mathrm{SKI}$ & 1.04 & 1302 & $5460-7430$ & 52190.6 & $-1.1 \pm 0.1$ & -0.04 \\
\hline 22-10-2001 & $1.9 \mathrm{~m} / \mathrm{OHP}$ & 0.44 & 1200 & $6250-7150$ & 52205.6 & $-1.0 \pm 0.1$ & 0.03 \\
\hline 05-12-2001 & $2.5 \mathrm{~m} / \mathrm{NOT}$ & 1.5 & 600 & $3800-6800$ & 52248.5 & $-1.4 \pm 0.1$ & -0.02 \\
\hline 20-01-2002 & $1.5 \mathrm{~m} / \mathrm{OHP}$ & 0.22 & 600 & $6380-6830$ & 52295.2 & $-1.9 \pm 0.1$ & -0.04 \\
\hline 25-07-2002 & $2.5 \mathrm{~m} / \mathrm{ORM}$ & 0.8 & 1200 & $6270-7120$ & 52480.7 & $-1.7 \pm 0.1$ & -0.04 \\
\hline 10-09-2002 & $1.3 \mathrm{~m} / \mathrm{SKI}$ & 1.04 & 1302 & $4680-6750$ & 52527.6 & $-1.5 \pm 0.1$ & 0.01 \\
\hline 28-10-2002 & $2.5 \mathrm{~m} / \mathrm{ORM}$ & 1.4 & 400 & $3400-7400$ & 52575.7 & $-2.0 \pm 0.1$ & -0.04 \\
\hline 06-10-2003 & $1.3 \mathrm{~m} / \mathrm{SKI}$ & 1.04 & 1302 & $5260-7330$ & 52918.6 & $-6.1 \pm 0.2$ & -0.03 \\
\hline 02-02-2004 & $4.2 \mathrm{~m} / \mathrm{ORM}$ & 0.22 & 1200 & $6100-6800$ & 53038.4 & $-6.0 \pm 0.2$ & 0.01 \\
\hline 25-08-2004 & $1.3 \mathrm{~m} / \mathrm{SKI}$ & 1.04 & 1302 & $4770-6840$ & 53243.6 & $-6.1 \pm 0.2$ & 0.03 \\
\hline 27-08-2004 & $1.3 \mathrm{~m} / \mathrm{SKI}$ & 1.04 & 1302 & $4770-6840$ & 53245.5 & $-6.0 \pm 0.2$ & -0.03 \\
\hline 24-10-2004 & $1.3 \mathrm{~m} / \mathrm{SKI}$ & 1.04 & 1302 & $4770-6840$ & 53303.4 & $-5.9 \pm 0.2$ & 0.00 \\
\hline $25-10-2004$ & $1.3 \mathrm{~m} / \mathrm{SKI}$ & 1.04 & 1302 & $4770-6840$ & 53304.6 & $-5.9 \pm 0.2$ & -0.03 \\
\hline 19-02-2005 & $4.0 \mathrm{~m} / \mathrm{KPNO}$ & 1.74 & 316 & 4940-9290 & 53420.7 & $-5.8 \pm 0.2$ & -0.01 \\
\hline \multicolumn{8}{|c|}{ Blue-end spectra } \\
\hline Date & $\begin{array}{l}\text { Telescope/ } \\
\text { Observatory }\end{array}$ & $\begin{array}{c}\text { Dispersion } \\
\AA ̊ ̊ \\
\text { Ápixel }\end{array}$ & $\begin{array}{c}\text { Grating } \\
1 / \mathrm{mm}\end{array}$ & $\begin{array}{c}\text { Wavelength } \\
\AA\end{array}$ & MJD & $\begin{array}{c}E W(\mathrm{H} \beta) \\
\AA\end{array}$ & profile \\
\hline 01-03-1996 & $1.0 \mathrm{~m} / \mathrm{ORM}$ & 0.44 & 1200 & $4550-5000$ & 50143.5 & -0.5 & shell \\
\hline 24-10-1997 & $1.0 \mathrm{~m} / \mathrm{ORM}$ & 0.90 & 1200 & $4000-4950$ & 50745.7 & -0.4 & shell \\
\hline 22-07-2000 & $1.3 \mathrm{~m} / \mathrm{SKI}$ & 1.04 & 1302 & $3800-5700$ & 51747.6 & +0.6 & absorption \\
\hline $17-10-2000$ & $1.3 \mathrm{~m} / \mathrm{SKI}$ & 1.04 & 1302 & $3600-5600$ & 51834.6 & +0.7 & absorption \\
\hline 09-08-2001 & $1.3 \mathrm{~m} / \mathrm{SKI}$ & 1.04 & 1302 & $3900-5900$ & 52130.6 & +1.6 & absorption \\
\hline 18-09-2001 & $1.5 \mathrm{~m} / \mathrm{OHP}$ & 0.22 & 600 & $4440-4900$ & 52170.7 & +1.7 & absorption \\
\hline 07-10-2001 & $1.3 \mathrm{~m} / \mathrm{SKI}$ & 1.04 & 1302 & $3900-5900$ & 52189.6 & +1.6 & absorption \\
\hline 23-10-2001 & $1.9 \mathrm{~m} / \mathrm{OHP}$ & 0.9 & 600 & $3745-5575$ & 52205.5 & +2.0 & absorption \\
\hline $25-10-2002$ & $2.5 \mathrm{~m} / \mathrm{ORM}$ & 0.5 & 1200 & $3600-5400$ & 52572.7 & +1.4 & absorption \\
\hline 02-02-2004 & $4.2 \mathrm{~m} / \mathrm{ORM}$ & 0.22 & 1200 & $4000-4800$ & 53038.4 & - & - \\
\hline
\end{tabular}

${ }^{a} V / R=\left(I(V)-I_{\mathrm{c}}\right) /\left(I(R)-I_{\mathrm{c}}\right)$.

\section{Observations}

\subsection{Optical observations}

Optical spectroscopic observations were obtained from 7 telescopes at 4 different observatories: from the Roque de los Muchachos observatory (ORM) in La Palma (Spain), observations were made with the $1.0 \mathrm{~m}$ Jacobus Kapteyn Telescope, the $2.5 \mathrm{~m}$ Isaac Newton Telescope, the $4.2 \mathrm{~m}$ William Herschel Telescope (service time) and the $2.5 \mathrm{~m}$ Nordic Optical
Telescope; from the Skinakas observatory (SKI) in Crete (Greece) the data come from the $1.3 \mathrm{~m}$ telescope; and from the Haut Provence observatory (OHP) in France the $1.52 \mathrm{~m}$ and the $1.93 \mathrm{~m}$ telescopes were employed. Finally one spectrum was taken from the $4 \mathrm{~m}$ telescope of the Kitt Peak National Observatory (KPNO) in the USA. Table 1 gives the log of the spectroscopic observations. This table contains instrumental information together with the results of the spectral analysis: the equivalent width of the $\mathrm{H} \alpha$ and $\mathrm{H} \beta$ lines and an indication of the profile shape of the lines. Negative values indicate that the 
Table 2. Optical and infrared magnitudes of LS V +44 17.

\begin{tabular}{|c|c|c|c|c|c|c|}
\hline \multicolumn{7}{|c|}{ Optical } \\
\hline Date & MJD & Observatory & $y$ & $b$ & $v$ & $u$ \\
\hline \multirow[t]{2}{*}{ 16-08-1999 } & 51407.50 & $1.3 \mathrm{~m} / \mathrm{SKI}$ & $10.82 \pm 0.03$ & $11.38 \pm 0.03$ & $11.64 \pm 0.05$ & $12.13 \pm 0.06$ \\
\hline & & & $B$ & $V$ & $R$ & $I$ \\
\hline 24-08-2004 & 53242.59 & $1.3 \mathrm{~m} / \mathrm{SKI}$ & $11.48 \pm 0.02$ & $10.85 \pm 0.02$ & $10.40 \pm 0.02$ & $9.92 \pm 0.02$ \\
\hline 14-09-2004 & 53263.56 & $1.3 \mathrm{~m} / \mathrm{SKI}$ & $11.39 \pm 0.02$ & $10.75 \pm 0.02$ & $10.30 \pm 0.02$ & $9.83 \pm 0.02$ \\
\hline 01-10-2004 & 53280.51 & $1.3 \mathrm{~m} / \mathrm{SKI}$ & $11.40 \pm 0.02$ & $10.76 \pm 0.02$ & $10.33 \pm 0.02$ & $9.85 \pm 0.02$ \\
\hline \multicolumn{7}{|c|}{ Infrared } \\
\hline Date & MJD & Instrument $^{a}$ & $J$ & $H$ & $K$ & \\
\hline $14-10-95$ & 50005.71 & CVF & $8.99 \pm 0.01$ & $8.73 \pm 0.01$ & $8.44 \pm 0.01$ & \\
\hline $12-01-96$ & 50095.58 & $\mathrm{CVF}$ & $9.06 \pm 0.01$ & $8.76 \pm 0.01$ & $8.49 \pm 0.01$ & \\
\hline $27-10-98$ & 51114.62 & $\mathrm{CVF}$ & $9.53 \pm 0.01$ & $9.47 \pm 0.04$ & $9.21 \pm 0.01$ & \\
\hline $27-10-98$ & 51114.63 & CVF & $9.60 \pm 0.01$ & $9.40 \pm 0.02$ & $9.26 \pm 0.01$ & \\
\hline $28-10-98$ & 51115.61 & $\mathrm{CVF}$ & $9.40 \pm 0.01$ & $9.18 \pm 0.01$ & $8.99 \pm 0.01$ & \\
\hline 02-10-99 & 51454.59 & CVF & $9.40 \pm 0.02$ & $9.21 \pm 0.02$ & $8.99 \pm 0.02$ & \\
\hline 22-01-00 & 51566.48 & CVF & $9.51 \pm 0.03$ & $9.36 \pm 0.02$ & $9.18 \pm 0.02$ & \\
\hline $22-01-00$ & 51566.49 & CVF & $9.56 \pm 0.02$ & $9.30 \pm 0.01$ & $9.23 \pm 0.02$ & \\
\hline $17-10-00$ & 51835.73 & $\mathrm{CVF}$ & $9.57 \pm 0.03$ & $9.45 \pm 0.02$ & $9.34 \pm 0.03$ & \\
\hline $11-01-01$ & 51921.51 & $\mathrm{CVF}$ & $9.57 \pm 0.03$ & $9.46 \pm 0.02$ & $9.37 \pm 0.02$ & \\
\hline $14-01-01$ & 51924.48 & CVF & $9.56 \pm 0.02$ & $9.40 \pm 0.02$ & $9.33 \pm 0.03$ & \\
\hline $23-03-02$ & 52357.40 & CAIN-II & $9.47 \pm 0.03$ & $9.18 \pm 0.03$ & $9.07 \pm 0.03$ & \\
\hline $08-11-02$ & 52587.78 & CAIN-II & $9.10 \pm 0.03$ & $8.89 \pm 0.03$ & $8.73 \pm 0.03$ & \\
\hline $09-11-02$ & 52588.67 & CAIN-II & $9.15 \pm 0.03$ & $8.92 \pm 0.03$ & $8.77 \pm 0.03$ & \\
\hline $12-11-02$ & 52591.54 & CAIN-II & $9.26 \pm 0.03$ & $8.93 \pm 0.03$ & $8.75 \pm 0.03$ & \\
\hline $13-11-02$ & 52592.67 & CAIN-II & $9.28 \pm 0.03$ & $8.95 \pm 0.03$ & $8.79 \pm 0.03$ & \\
\hline $30-08-04$ & 53248.69 & CAIN-II & $9.24 \pm 0.03$ & $8.83 \pm 0.03$ & $8.71 \pm 0.03$ & \\
\hline 29-12-04 & 53369.57 & FIN & $9.25 \pm 0.10$ & $9.03 \pm 0.03$ & $8.72 \pm 0.04$ & \\
\hline $30-12-04$ & 53370.57 & FIN & $9.19 \pm 0.04$ & $9.03 \pm 0.03$ & $8.73 \pm 0.04$ & \\
\hline
\end{tabular}

${ }^{a}$ All observations were obtained from the 1.5-m Carlos Sánchez Telescope.

line is in emission. The reduction of the spectra was made using the STARLINK Figaro package (Shortridge et al. 2001), while their analysis was performed using the STARLINK Dipso package (Howarth et al. 1998).

Optical photometric observations were made using two photometric systems. Strömgren photometry (uvby) was obtained from the Skinakas observatory (SKI) on August 16, 1999. LS V +44 17 was also observed through the Johnson $B, V, R$ and $I$ filters on three occasions from the Skinakas observatory (see Table 2). The telescope was equipped with a $1024 \times 1024$ SITe CCD chip, containing $24 \mu \mathrm{m}$ pixels. Reduction of the data was carried out using the IRAF tools for aperture photometry.

\subsection{Infrared observations}

Infrared photometry in the $J H K$ bands was obtained as part of a monitoring programme of Be/X-ray binaries at the $1.5 \mathrm{~m}$. Carlos Sánchez Telescope (TCS), located at the Teide Observatory in Tenerife, Spain. The instruments used were the Continuously Variable Filter Photometer (CVF) up to January 2001, and the CAIN-II camera, equiped with a $256 \times$ $256 \mathrm{HgCdTe}$ (NICMOS 3) detector ever since. The last data in December 2004 were obtained with the recently comissioned FIN photometer.

Instrumental CVF and FIN magnitudes were transformed to the standard system defined by Kidger \& Martín-Luis (2003). Instrumental CAIN magnitudes were obtained from the images by means of the IRAF tools for aperture photometry, and transformed to the standard system defined by Hunt et al. (1998). The accuracy of the standard $J H K$ values in all three bands is $0.01,0.03$ and $0.02 \mathrm{mag}$. for CVF, CAIN-II and FIN data respectively. The obtained values are given in Table 2.

\section{Results}

\subsection{Previous optical work}

The first astronomical observations date back to the circa 1930. LS V +44 17 is mentioned in the Bergedorfer SpektralDurchmusterung catalogue (BSD, Schwassmann \& van Rhijn 1935), where a spectral type B0 is suggested. The first 


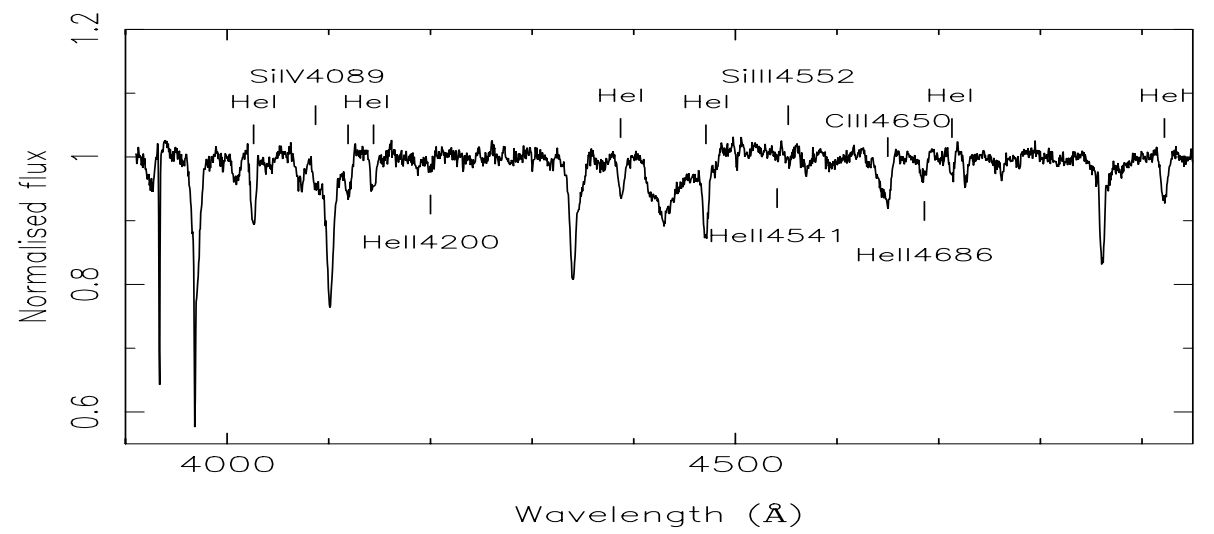

Fig. 1. Blue spectrum showing the lines used for spectral classification. The spectrum was taken from the INT on October 25, 2002. The He I lines marked are $\lambda 4026, \lambda 4121, \lambda 4144, \lambda 4387, \lambda 4471, \lambda 4713$ and $\lambda 4921$.

accurate photometric observations on the Johnson photometric system were performed by Bigay (1963) who gave $V=10.78$, $(B-V)=0.61$ and $(U-B)=-0.36$ for observations performed in 1953, although the star is referenced in Seyfert \& Popper (1941) with a photographic magnitude of 11.3. LS V +44 17 is also found on one of the photographic plates of the Sandage two-colour survey of the Galactic plane (Lanning \& Meakes 2001) with $B=10.4$ and $(U-B)=-0.4$, although these val-

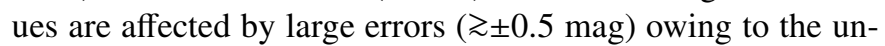
certainties inherent to obtaining accurate visual estimates from photographic plates.

Further photometric data are provided by catalogues of optical surveys. However, varying results are obtained for different versions of the catalogue due to slightly different reduction methods. Another disadvantage of the catalogued values is the difficulty in defining the exact date of the observations. The Hipparcos and Tycho Catalogues (Hog et al. 2000) give $V=10.71$ and $(B-V)=0.44$ for observations that took place some time from November 1989 to March 1993, whereas the USNO-A2.0 Catalogue (Monet et al. 1998) gives a blue magnitude of 12.2 and a red magnitude of 10.4 for the epoch 1953.025.

\subsection{Spectral classification}

The presence of $\mathrm{H}, \mathrm{He}$ I and Si IV lines clearly demonstrates that RX J0440.9+4431 contains an early-type star (Fig. 1). He II lines, although present, are weak (only He II $4686 \AA$ is clearly detected, He II $4200 \AA$ is weak and He II $4541 \AA$ is buried in the noise), which implies that the spectral type must be earlier than B1 but later than 09.5. A spectral type in this range is also borne out by the weak Si III $4552 \AA$. He II $4686 \AA$ is normally last seen at B0.5, whereas the ratio Si III $4552 \AA /$ Si IV $4089 \AA$ increases smoothly as we progress toward later spectral types. A visual comparison of the relative strength of metallic lines of LS V +44 17 with those of MK standards (Walborn \& Fitzpatrick 1990) resulted in B0.2 as the closest spectral type. In particular, the fact that the ratio of the C III $4650 \AA$ blend with respect to He II $4686 \AA$ is larger than one agrees more with a B0.2 than with a B0 type. As for the luminosity class, a main-sequence star is suggested

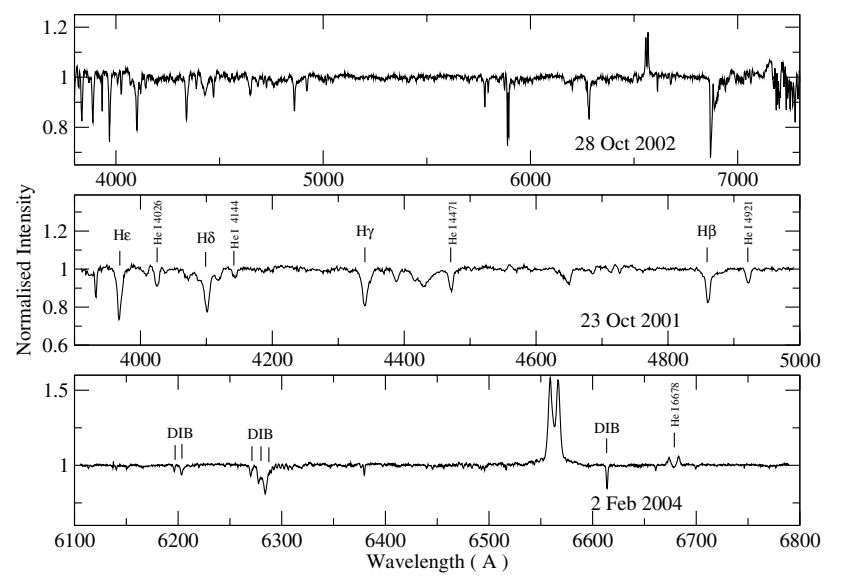

Fig. 2. Top: broad-band low-resolution spectrum of the optical companion of RX J0440.9+4431. Middle and bottom: blue-end and redend spectra obtained with higher resolution. The most relevant hydrogen and helium lines and diffuse interstellar bands are marked. The Balmer series can be seen as well.

by the fact that the ratios of He I $4026 \AA$ over Si IV $4089 \AA$ and He I $4121 \AA$ over Si IV $4116 \AA$ are larger than one. Therefore, we conclude that the optical counterpart to the $\mathrm{Be} / \mathrm{X}$-ray binary RX J0440.9+4431 is a B0.2V star.

\subsection{Reddening and distance}

Besides the $\mathrm{H} \alpha$ and $\mathrm{He} \mathrm{I} \lambda 6678$ lines, the red-end spectrum (Fig. 2) contains several strong diffuse interstellar bands (DIB), which can be used to estimate the amount of interstellar absorption toward the source (Herbig \& Leka 1991; Galazutdinov et al. 2000). We used six different interstellar lines (6010 , $6195 \AA, 6202 \AA, 6269 \AA, 6376 / 79 \AA$ and $6613 \AA$ ) to derive a colour excess $E(B-V)$ according to the linear relationships of Herbig (1975). Given the different amount of available data (the spectra covered different wavelength intervals) and different signal-to-noise values of the spectra, we obtained a mean $E(B-V)$ by averaging all measurements. The resulting reddening was $E(B-V)=0.62 \pm 0.03$. For the sake of comparison, we also obtained the colour excess using only the average of the five measurements of the highest resolution spectra 
(those taken from the WHT on February 2, 2004). The resulting $E(B-V)$ was $0.64 \pm 0.02$. The errors are the weighted standard deviation of the results of the various lines used.

Estimating the reddening from photometric data in Be stars might be misleading as the circumstellar continuum emission affects the photometric colours and indices (Fabregat \& Torrejón 1998). This effect is expected to be more distinct for longer wavelengths. Indeed, a B0.2V star has an intrinsic colour $(B-V)_{0}=-0.25$ (Wegner 1994). Taking the measured photometric magnitudes $(B-V)=0.63$ (2004 observations) we derive an excess $E(B-V) \approx 0.9$, somewhat larger than the value derived above. However, LS V +44 17 went through a lowactivity optical state, presumably during the first half of 2001 as indicated by the low $\mathrm{H} \alpha$ equivalent widths (see Fig. 5). Interpreting this optical minimum as a weakening of the disc we would expect that the IR magnitudes at that time should be very close to those of the underlying $B$ star. Assuming the interstellar extinction law $E(J-K)=0.54 E(B-V)$ and the intrinsic colour $(J-K)_{0}=-0.16$ for a main-sequence B0.2 star (Koornneef 1983), the observed $(J-K)=0.21$ gives $E(B-V)=0.68 \pm 0.03$, in good agreement with the spectroscopically derived value.

Finally, taking the standard law $A_{V}=3.1 E(B-V)$ and assuming an average absolute magnitude for a $\mathrm{B} 0.2 \mathrm{~V}$ star of $M_{V}=-3.8$ (Humphreys \& McElroy 1984; Martins et al. 2005) the distance to RX J0440.9+4431 is estimated to be $\sim 3.3 \pm$ $0.5 \mathrm{kpc}$. This error includes those of $m_{V}(0.02)$ and $A_{V}(0.3)$, but assumes no error in the absolute magnitude $M_{V}$.

\subsection{Rotational velocity}

The rotational velocity was estimated by measuring the full width at half maximum of He I lines (see e.g. Steele et al. 1999). After correcting for instrumental resolution we obtained $v \sin i=235 \pm 15 \mathrm{~km} \mathrm{~s}^{-1}$, which compares favourably to the value of $246 \pm 16 \mathrm{~km} \mathrm{~s}^{-1}$ given for weak-emission earlytype shell stars (Mennickent et al. 1994). As a comparison, other rotational velocities in Be/X-ray binaries are: $v \sin i=$ $200 \pm 30 \mathrm{~km} \mathrm{~s}^{-1}$ in LS I +61 235/RX J0146.9+6121 (Reig et al. 1997a), $v \sin i=290 \pm 50 \mathrm{~km} \mathrm{~s}^{-1}$ in V635 Cas/4U 0115+63 (Negueruela \& Okazaki 2001), $v \sin i=240 \pm 20 \mathrm{~km} \mathrm{~s}^{-1}$ in LS 992/RX J0812.4-3114 (Reig et al. 2001), $v \sin i=240 \pm$ $20 \mathrm{~km} \mathrm{~s}^{-1}$ in SAX J2103.5+4545 (Reig et al. 2004).

\section{Discussion}

We have monitored the Be/X-ray binary LS V +44 17 for the last 10 years. Our observations coincided with the latest stages of a declining disc phase. The slow and gradual decline of the $E W(\mathrm{H} \alpha)$ and IR colours seems to indicate that the mechanism that feeds the disc had already stopped when we started the monitoring of the source. The source entered a long period (1998-2003) of low optical/IR activity, where the line emission just filled in the underlying absorption expected from the photosphere of the B-type star and the IR magnitudes showed their lowest values. The equivalent width of the $\mathrm{H} \alpha$ line $(E W(\mathrm{H} \alpha))$ always remained negative, indicating that the complete loss of the disc did not occur. However, given the large observational gaps of our data we cannot rule out the possibility that such an event could have happened. The loss of the circumstellar disc could have occurred in early 2001 (the $E W(\mathrm{H} \alpha)$ was only $-0.2 \AA$ in August 2001). As mentioned before, in January 2001 the measured intrinsic IR colour $(J-K) \approx-0.16$ agrees with a B0-B0.5 star (Koornneef 1983). In other words, in 2001 the underlying B-type star would have been exposed. Figure 5 shows the evolution of the $\mathrm{H} \alpha$ equivalent width and the infrared magnitudes. The $E W(\mathrm{H} \alpha)$ and the IR magnitudes follow the same trend, namely, a slow decrease, reaching a minimum around MJD 52000 and a gradual increase. This longterm variability suggests the dissipation and subsequent formation of the circumstellar disc and sets a common origin (i.e., the circumstellar disc) for the $\mathrm{H} \alpha$ emission and infrared excess.

\subsection{The $H \alpha$ line}

$V / R$ variability is defined as the intensity variations of the two peaks (known as violet and red peak) in the split profile of a spectral line. In many Be stars, if monitored over a long enough periods of time, these variations are quasiperiodic (Okazaki 1997). The $V / R$ ratio is defined as $V / R=\left(I(V)-I_{\mathrm{c}}\right) /\left(I(R)-I_{\mathrm{c}}\right)$, where $I(V), I(R)$ and $I_{\mathrm{c}}$ are the intensities of the violet peak, red peak and continuum, respectively.

The evolution of the $\mathrm{H} \alpha$ profile throughout the period covered by our observations is presented in Fig. 3. The vertical scale was left the same in all plots in order to show the variability in the strength of the line. Double-peak $\mathrm{H} \alpha$ line profiles, both symmetric and asymmetric, are always present in LS V +44 17. Symmetric profiles are believed to be generated in quasi-Keplerian discs (see e.g. Hummel 1994). Asymmetric profiles are associated with radial motion and/or distorted density distributions (Hanuschik et al. 1995; Hummel \& Hanuschik 1997). The model that most successfully accounts for the long-term variability of these asymmetric profiles is the one-armed oscillation model (Okazaki 2000, and references therein).

In LS V +44 17, the ratio of the intensities of the violet over the red peak $(V / R)$ hardly varied over almost 10 years. Excluding the first two spectra, the lines have $|\log (V / R)|<$ 0.05 (Col. 8 of Table 1). A symmetric profile does not necessarily mean the absence of the density wave as symmetric split profiles (the $V=R$ phase) can occur during a fraction of the $V / R$ cycle, more precisely, when the star lies between the observer and the high-density perturbation (Telting et al. 1994). These $V=R$ phases represent a fraction of the entire $V / R$ cycle. In LS V +44 17 an asymmetric profile was last seen in 1996. Since then only symmetric profiles are present. Thus it is very unlikely that the spectral state of the last 8 years correspond to a $V / R$ phase. We conclude that the density wave faded away before the dissipation of the disc, perhaps because the disc became too tenuous to support a density wave.

Some spectra show the depression between the double peak profile extending below the stellar continuum, reminiscent of the shell profile. These types of lines are explained by partial absorption of the central star by the circumstellar disc as 

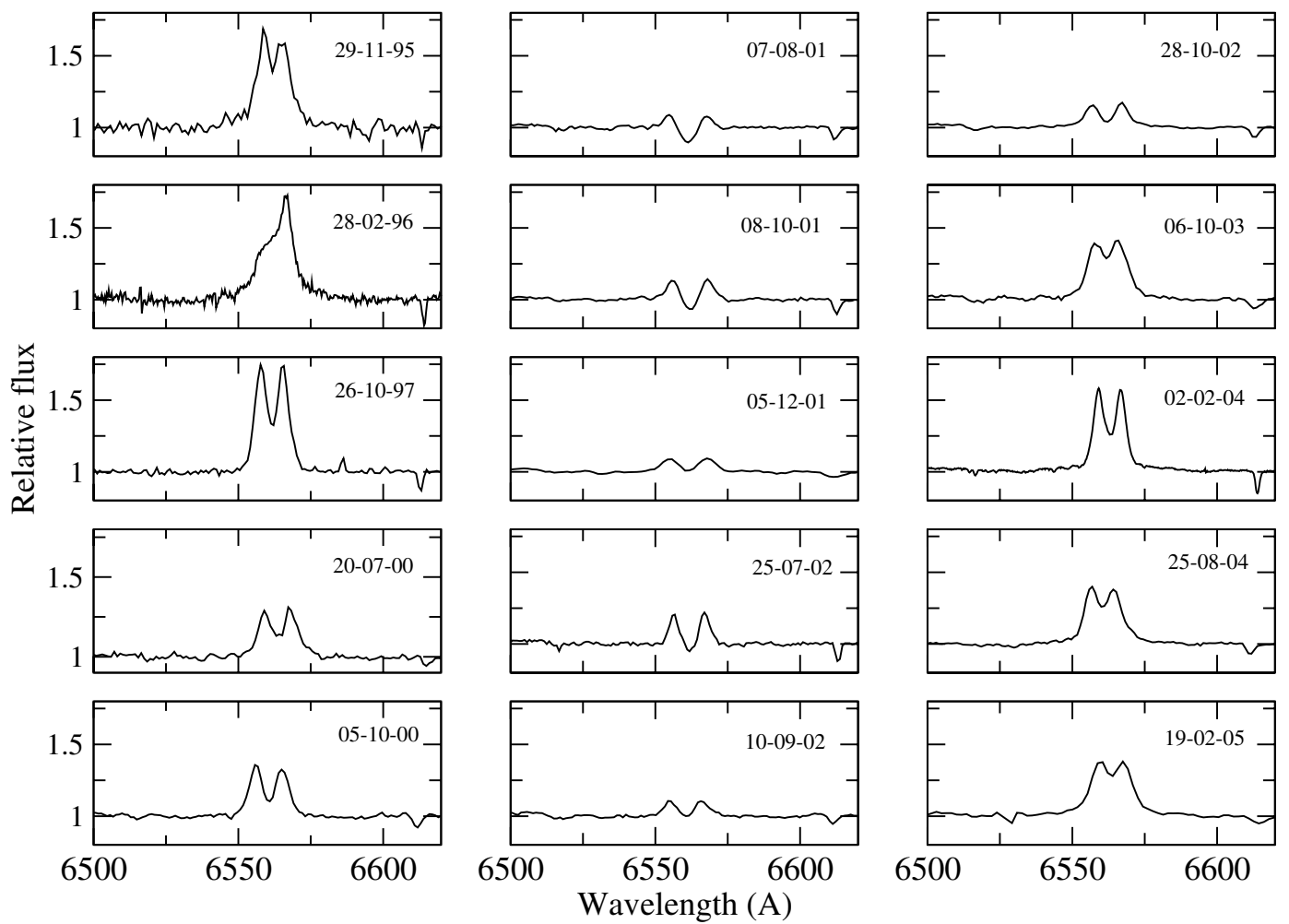

Fig. 3. Evolution of the $\mathrm{H} \alpha$ line profile for the past 10 years. See Table 1 for the complete set of observations.

a consequence of a high inclination angle (see e.g. Hummel \& Vrancken 2000). However, the profiles seen in LS V +44 17 cannot be considered as proper shell lines because they only occurred during the optical minimum. If ascribed to absorption by the disc itself then we should expect the central depression to become more apparent as the extent of the disc increases, i.e., as the $E W(\mathrm{H} \alpha)$ increases. No such trend is seen (Fig. 4). In addition, the width of the central reversal is considerably broader $\left(F W H M \approx 200-400 \mathrm{~km} \mathrm{~s}^{-1}\right)$ than the typical value of shell profiles $\left(F W H M \lesssim 50 \mathrm{~km} \mathrm{~s}^{-1}\right)$. Finally, none of the spectra of LS V +44 17 fulfil the criterion given by Hanuschik (1996) that in order for a profile to have shell characteristics the ratio $I_{\mathrm{p}} / I_{\mathrm{cd}}$ should be larger than 1.5 . Therefore the apparent shell profiles in LS V +44 17 are likely to be due to the photospheric absorption line, which combines with a weak double-peaked emission.

Significant changes are apparent in the distance between the peaks and the strength of the line. The peak separation correlates with the intensity of the $\mathrm{H} \alpha$ line (Fig. 4). As the $E W(\mathrm{H} \alpha)$ increases, the distance between peaks decreases. Interpreting the peak separation $\left(\Delta_{\text {peak }}\right)$ as the outer radius $\left(R_{\text {out }}\right)$ of the emission line forming region (Huang 1972)

$\frac{R_{\text {out }}}{R_{*}}=\sqrt{\frac{2 v \sin i}{\Delta_{\text {peak }}}}$

we conclude that lower velocities of the emitting components occur when the disc has developed, i.e., when the $E W(\mathrm{H} \alpha)$ is large. Despite a moderate increase of the $E W(\mathrm{H} \alpha)$ the absence of asymmetries indicates that fast radial displacements do not take place during the first instances of the formation of the disc.

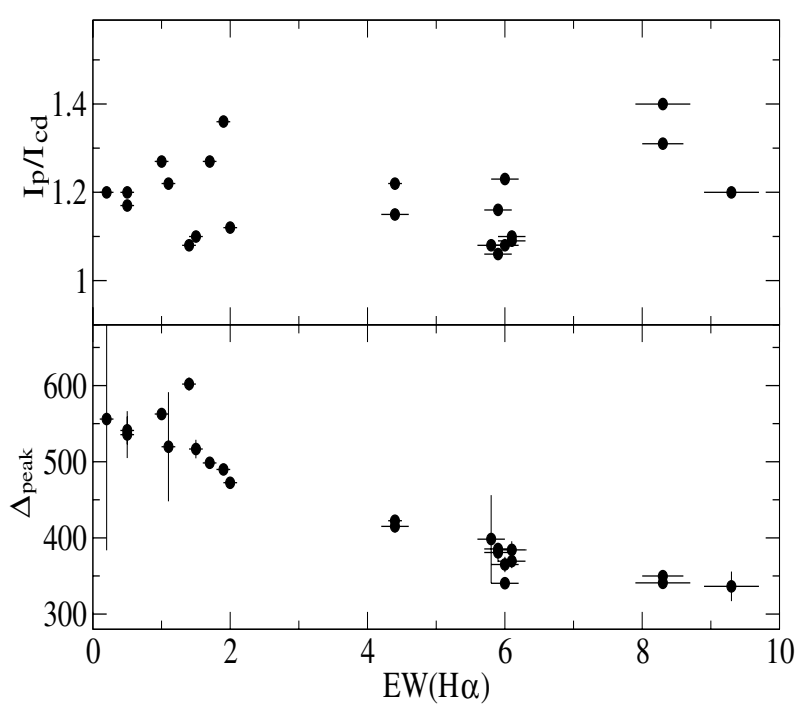

Fig. 4. Top: mean intensity of the blue and red peaks $I_{\mathrm{p}}$ over that of the central reversal $I_{\mathrm{cd}}$. Bottom: peak separation as a function of the $\mathrm{H} \alpha$ equivalent width. Note that the spectral resolution constitutes a source of scattering in these diagrams.

The He I $6678 \AA$ line also shows $V / R$ variability. In general, it imitates the behaviour of the $\mathrm{H} \alpha$ line. Since metallic lines are generated at smaller disc radii than the hydrogen lines (Hummel \& Vrancken 1995; Jaschek \& Jaschek 2004), the asymmetry of the He I line profiles indicates that the internal changes of the disc are global, affecting its entire structure. 
Table 3. Typical time scales for disc variability in Be/X-ray binaries.

\begin{tabular}{llccccl}
\hline \hline $\begin{array}{l}\text { X-ray } \\
\text { name }\end{array}$ & $\begin{array}{l}\text { Optical } \\
\text { name }\end{array}$ & $\begin{array}{c}\text { Spectral } \\
\text { type }\end{array}$ & $\begin{array}{c}T_{\text {disc }} \\
\text { (year) }\end{array}$ & $\begin{array}{c}T_{V / R} \\
\text { (year) }\end{array}$ & $\begin{array}{c}P_{\text {orb }} \\
\text { (day) }\end{array}$ & Reference \\
\hline 4U 0115+63 & V635 Cas & B0.2Ve & $3-5$ & $0.5-1.5$ & 24.3 & Negueruela \& Okazaki (2001) \\
V 0332+53 & BQ Cam & O9Ve & $4-5$ & 1 & 34.2 & Negueruela et al. (1999); Goranskii (2001) \\
4U 0352+309 & X Per & B0Ve & 7 & $0.6-2$ & 250 & Clark et al. (2001) \\
1A 0535+262 & V725 Tau & O9.7IIIe & $4-5$ & $1-1.5$ & 111 & Haigh et al. (2004); Clark et al. (1998) \\
RX J0812.4-3114 & LS 992 & B0.2IVe & 4 & - & 80 & Reig et al. (2001) \\
RX J 0146.9+6121 & LS I +61 235 & B1Ve & $>10$ & 3.4 & $>200^{*}$ & Reig et al. (2000) \\
4U 1145-619 & V801 Cen & B1Ve & $>10$ & 3 & 186 & Stevens et al. (1997) \\
RX J0440.9+4431 & LS V +44 17 & B0.2Ve & $>10$ & - & $>150^{*}$ & this work \\
\hline
\end{tabular}

* Obtained from the $P_{\text {spin }}-P_{\text {orb }}$ correlation (Corbet 1986).
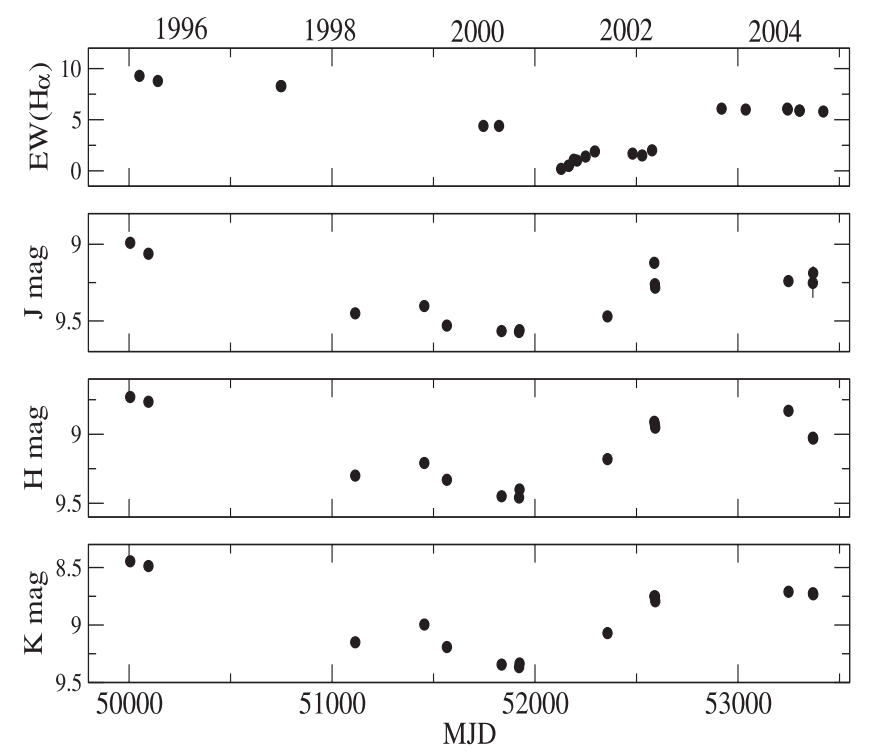

Fig. 5. Evolution of the $\mathrm{H} \alpha$ equivalent width and IR magnitudes for the past 10 years. Errors are included in the size of the points.

\subsection{Variability time scales}

Although the spectroscopic data are distributed irregularly over the period of the reported observations, the smooth variations of the $\mathrm{H} \alpha$ equivalent width and infrared brightness indicate that structural changes in the circumstellar disc of LS V +44 17 occur on time scales of years.

Table 3 shows the typical time scales associated with disc variability for a number of $\mathrm{Be} / \mathrm{X}$-ray binaries: $T_{\text {disc }}$ is the typical duration of formation/dissipation of the circumstellar disc and $T_{V / R}$ represents the quasi period for $V / R$ variability. $T_{\text {disc }}$ exhibits a good correlation with the orbital period. Systems with narrow orbits tend to show faster disc growth and dissipation cycles, while slower evolutionary time scales are associated with long orbital periods. This is in agreement with the disc truncation model (Okazaki \& Negueruela 2001), which suggests a direct relationship between the size of the disc and the orbital period (see also Reig et al. 1997b, for observational evidence in this respect). Within the framework of the global one-armed oscillation model, the viscous excitation of a density wave is associated with longer time scales when the disc is larger (Okazaki 2000).

Although the orbital period of RX J0440.9+4431/ LS V +44 17 is unknown, its classification as a persistent system (Reig \& Roche 1999) and its relatively long spin period imply that it must be long. A $P_{\text {orb }}=150-200 \mathrm{~d}$ is estimated from the $P_{\text {spin }}-P_{\text {orb }}$ diagram (Corbet 1986). The typical time scales associated with the evolution of the circumstellar disc in LS V +44 17 are rather long - the $E W(\mathrm{H} \alpha)$ had not recovered from the initial pre-disc-loss phase values nine years later - and agree with those of Be/X-ray binaries with wide orbits. In contrast, changes originated by the density wave are much faster. In LS V +44 17, the time elapsed between the slightly blue-dominated line of the first spectrum and the strongly red-dominated line of the second spectrum of Fig. 3 is just 3 months.

None of the three Be/X-ray binaries that have gone through disc-loss phases and for which there is a good optical followup coverage, namely, X Per in 1990 (Clark et al. 2001), 4U 0115+63 in 1997 (Negueruela \& Okazaki 2001) and 1A 0535+262 in 1998 (Haigh et al. 2004), exhibited asymmetric profiles during the initial stage of disc growth. After the disc loss phase the first asymmetric profile did not occur until the $E W(\mathrm{H} \alpha)$ was $\sim 6-7 \AA$ in $4 \mathrm{U} 0115+63\left(P_{\text {orb }}=24.3 \mathrm{~d}\right)$, $\sim 7-10 \AA$ in $1 \mathrm{~A} 0535+262\left(P_{\text {orb }}=111 \mathrm{~d}\right)$ and $\sim 10-12 \AA$ in $\mathrm{X} \operatorname{Per}\left(P_{\text {orb }}=250 \mathrm{~d}\right)$. In LS V +44 17/RX J0440.9+4431, asymmetric profiles are associated with the largest values of the $E W(\mathrm{H} \alpha)$. Below $8 \AA$, only symmetric profiles are observed. The peak separation of the latest spectra imply a disc radius of $\sim 2 R_{*}$, assuming a Keplerian disc and Eq. (1).

In summary, the correlation of $T_{\text {disc }}$ and the orbital period provides further observational evidence for the interaction of the neutron star with the circumstellar disc of its Be star's companion, whilst the relationship betwen the $E W(\mathrm{H} \alpha)$ at which the first asymmetry appears with the orbital period implies that the density oscillations do not become observable until the disc has reached a critical size or density. 


\section{Conclusion}

We have monitored the Be/X-ray binary LS V +44 17 for the last 10 years. The observations coincided with a period of low optical/IR activity, characterised by the likely loss of the Be star's circumstellar disc and subsequent reformation. Since 2001 the envelope has been gradually growing as indicated by the increase of the equivalent width and the narrowing of the peak separation of the split $\mathrm{H} \alpha$ line. The time scales for structural changes in the circumstellar disc of RX J0440.9+4431/LS V +44 17 compares favourably with those of Be/X-ray binaries with long orbital periods. While the formation/dissipation of the disc may last for several years, the line profile changes are much faster and, in general, depends on the duration of the active phase. The disappearance of the $V / R$ varibility before the dissipation of the disc and the lack of asymmetric profiles of the latest observations even though the equivalent width of the $\mathrm{H} \alpha$ line has increased up to $\sim 6 \AA$ confirms the fact that the effects of the density perturbation do not manifest themselves until the disc is fully developed. By studying the characteristic variability time scales of a number of $\mathrm{Be} / \mathrm{X}$-ray binaries we have found further observational evidence of the influence of the neutron star on the envelope of the Be star.

Acknowledgements. The authors thank M. Brotherton and P. Nandra for providing the Kitt Peak spectrum. The Kitt Peak National Observatory, a division of the National Optical Astronomy Observatories, is operated by the Association of Universities for Research in Astronomy, Inc. under cooperative agreement with the National Science Foundation. I.N. is a researcher of the programme Ramón y Cajal, funded by the Spanish Ministerio de Educación y Ciencia and the University of Alicante, with partial support from the Generalitat Valenciana and the European Regional Development Fund (ERDF/FEDER). This research is partially supported by the MEC through grant ESP-2002-04124-C03-03. Skinakas Observatory is a collaborative project of the University of Crete, the Foundation for Research and Technology-Hellas and the Max-Planck-Institut für Extraterrestrische Physik. The WHT spectrum was obtained as part of the ING service programme. Based in part on observations made at Observatoire de Haute Provence (CNRS), France. The Carlos Sanchez Telescope is operated at the Teide Observatory by the Instituto de Astrofísica de Canarias.

\section{References}

Bigay, J. H. 1963, JO, 46, 319

Clark, J. S., Tarasov, A. E., Steele, et al. 1998, MNRAS, 294, 165

Clark, J. S., Tarasov, A. E., Okazaki, A. T., Roche, P., \& Lyuty, V. M. 2001, 380, 615

Corbet, R. H. D. 1986, MNRAS, 220, 1047

Fabregat, J., \& Torrejón, J. M. 1998, A\&A, 332, 643

Galazutdinov, G. A., Musaev, F. A., Krelowski, J., \& Walker, G. A. H. 2000, PASP, 112, 648

Goranskii, V. P. 2001, AstL, 27, 516

Haigh, N. J., Coe, M. J., \& Fabregat, J. 2004, MNRAS, 350, 1457
Hanuschik, R. W., Hummel, W., Dietle, O., \& Sutorius, E. 1995, A\&A, 300, 163

Hanuschik, R. W. 1996, A\&A, 308, 170

Herbig, G. H. 1975, ApJ, 196, 129

Herbig, G. H., \& Leka, K. D. 1991, ApJ, 382, 193

Hog, E., Fabricius, C., Makarov, V. V., et al. 2000, A\&A, 355, L27, VizieR On-line Data Catalog: I/259

Howarth, I. D., Murray, J., Mills, D., \& Berry, D. S. 1998, Starlink User Note, 50.21

Huang, S. S. 1972, ApJ, 171, 549

Hummel, W. 1994, A\&A, 289, 458

Hummel, W., \& Hanuschik, R. W. 1997, A\&A, 320, 852

Hummel, W., \& Vrancken, M. 1995, 302, 751

Hummel, W., \& Vrancken, M. 2000, 359, 1075

Humphreys, R. M., \& McElroy, D. B. 1984, ApJ, 284, 565

Hunt, L. K., Mannucci, F., Testi, L., et al. 1998, AJ, 115, 2594

Jaschek, C., \& Jaschek, M. 2004, yCat, 6057, 0. VizieR On-line Data Catalog: VI/57, Originally published in: 1993, A\&AS, 97, 807

Kidger, M. R., \& Martín-Luis, F. 2003, AJ, 125, 3311

Koornneef, J. 1983, A\&A, 128, 84

Landolt, A. U. 1992, AJ, 104, 340

Lanning, H. H., \& Meakes, M. 2001, PASP, 113, 1393

Martins, F., Schaerer, D., \& Hillier, D. J. 2005, A\&A, 436, 1049

Mennickent, R. E., Vogt, N., Barrera, L. H., Covarrubias, R., \& Ramirez, A. 1994, A\&AS, 106, 427

Monet, D., Bird, A., Canzian, et al. 1998, VizieR On-line Data Catalog: I/252

Motch, C., Haberl, F., Dennerl, K., Pakull, M., \& Janot-Pacheco, E. 1997, A\&A, 323, 853

Negueruela, I., Roche, P., Fabregat, J., \& Coe, M. J. 1999, MNRAS, 307,695

Negueruela, I., \& Okazaki, A. T. 2001, A\&A, 369, 108

Okazaki, A. T. 1997, A\&A, 318, 548

Okazaki, A. T. 2000, in The Be Phenomenon in Early-Type Stars, IAU Coll. 175, ASP Conf. Proc., 214, ed. M. A. Smith, \& H. F. Henrichs, 409

Okazaki, A. T., \& Negueruela, I. 2001, A\&A, 377, 161

Porter, J. M., \& Rivinius, T. 2003, PASP, 115, 1153

Reig, P., Fabregat, J., Coe, M. J., et al. 1997a, A\&A, 322, 183

Reig, P., Fabregat, J., \& Coe 1997b, A\&A, 322, 193

Reig, P., \& Roche, P. 1999, MNRAS, 306, 100

Reig, P., Negueruela, I., Coe, M. J., et al. 2000, MNRAS, 317, 205

Reig, P., Negueruela, I., Buckley, D. A. H., et al. 2001, A\&A, 367, 266

Reig, P., Negueruela, I., Fabregat, J., et al. 2004, A\&A, 421, 673

Seyfert, C. K., \& Popper, D. M. 1941, ApJ, 93, 461

Schwassmann, A., \& van Rhijn, P. J. 1935, Bergedorf: Hamburger Sternwarte

Shortridge, K., Meyerdierks, H., Currie, M., et al. 2001, Starlink User Note, 86.19

Steele, I. A., Negueruela, I., \& Clark, J. S. 1999, A\&AS, 137, 147

Stevens, J. B., Reig, P., Coe, M. J., et al. 1997, MNRAS, 288, 988

Telting, J. H., Heemskerk, M. H. M., Henrichs, H. F., \& Savonije, G. J. 1994, A\&A, 288, 558

Townsend, R. H. D., Owocki, S. P., \& Howarth, I. D. 2004, MNRAS, 350, 189

Walborn, N. R., \& Fitzpatrick, E. L. 1990, PASP, 102, 379

Wegner, W. 1994, MNRAS, 270, 229 